\title{
Consequences of using different pair-correlation functions on the stability properties of the Homogeneous Cooling State for a monodisperse system of near-elastic disks
}

\author{
Sebastián González and Stefan Luding \\ Multi Scale Mechanics, TS, CTW, UTwente, \\ P.O.Box 217, 7500 AE Enschede, Netherlands, \\ e-mail: j.s.l.gonzalezbriones@utwente.nl, s.luding@utwente.nl
}

\begin{abstract}
We show the differences in the stability properties of the Homogeneous Cooling State (HCS) of a two-dimensional monodisperse collection of rigid and near-elastic disks, obtained by using different formulae for the pair-correlation function.

For an equation of state that takes into account the crystallization and ordering of the particles (and the respective pressure drop), the critical wavelength of the heat conduction mode is considerably modified in the transition zone, involving a bifurcation and an additional mode of instability. The theoretical predictions, using the improved equation of state, are confirmed by numerical simulations. Nevertheless, some open questions remain.
\end{abstract}

\section{Introduction}

Structure formation in a granular gas has attracted much attention during the last decades (see for example, Refs. [1-10]). Starting from a macroscopically homogeneous system, structures evolve and a dilute granular gas coexists with denser, possibly much denser and even solid, clusters - in non-equilibrium. However, the coexistence of a fluid-like granular gas with a solidlike packing also occurs in many other systems, like during avalanche flow on inclined planes or in vibrated containers, see Refs. $[11,12]$ and references therein.

In the absence of walls and external forces, the crucial phenomena in a freely cooling granular gas involve the fluctuations in density, velocity and temperature, which cause positiondependent energy loss [2]. In denser areas, due to strong local dissipation, pressure and energy decay rapidly and material moves from 'hot' to 'cold' regions, there leading to even stronger dissipation and thus causing the density instability with ever growing (dense) clusters.

The freely cooling granular gas will be introduced first, as an example of a case where dilute and dense granular media co-exist. Even though the need to treat walls is avoided by using periodic boundary conditions, and the initial state is macroscopically homogeneous, resembling a classical, elastic hard sphere gas, the system develops interesting dynamics and structure formation - only due to the dissipative interactions of the particles, see Fig. 1 in section 2. Hydrodynamic equations and constitutive relations are introduced in section 3 and a stability analysis of these equations is presented in section 4 . This has been done several times in the literature (see for example Refs. $[2,3,13,14]$ ), but always for a given (Enskog) pair correlation function (at contact distance, $g(\nu ; r=d)$ ). Our contribution is to study the consequences of an empirical pair correlation function on the instabilities of the HCS. Section 5 provides concluding comments. 


\section{From homogeneous to inhomogeneous cooling}

When a homogeneous granular gas cools down due to collisional dissipation, one observes an initial homogeneous cooling state. Previous studies have shown that granular cooling results in the formation of structures: both mass and momentum density spontaneously become nonuniform. Four different regimes - kinetic, shearing, clustered, and collapsed - have been identified, (see Ref. [4] and references therein). For the clustered regime (the one we are interested in here), two stages with different energy dissipation behavior can be identified: a cluster growth regime that follows the homogeneous state, and a final (inhomogeneous, non-equilibrium) state, where the cluster size is comparable to the system size and the structures span the whole system $[2-4,6,15-18]$.

Using the hard sphere model and event driven simulations, see e.g. Ref. [19], it is straightforward to simulate the time-evolution of a homogeneous granular gas with density (area fraction) $\nu=0.25$, about $N=2000$ particles of diameter $\hat{d}$, mass $\hat{m}$, and moderate dissipation with a coefficient of restitution $r=0.9$.

\subsection{Collision Model}

For two particles, $p=1,2$, at positions $\mathbf{r}_{1,2}$, conservation of momentum leads to a collision rule:

$$
\mathbf{v}_{1,2}^{\prime}=\mathbf{v}_{1,2} \mp \frac{1+r}{2}\left(\hat{\mathbf{k}} \cdot\left(\mathbf{v}_{1}-\mathbf{v}_{2}\right)\right) \hat{\mathbf{k}}
$$

where the prime indicates the velocities $\mathbf{v}$ after the collision, and $\hat{\mathbf{k}}=\left(\mathbf{r}_{2}-\mathbf{r}_{1}\right) /\left|\mathbf{r}_{2}-\mathbf{r}_{1}\right|$ is the unit vector pointing along the line of centers, from particle 1 to particle 2 .

At collision, the normal component, $v_{n}=\hat{\mathbf{k}} \cdot\left(\mathbf{v}_{1}-\mathbf{v}_{2}\right)$, of the relative velocity, $\mathbf{v}_{1}-\mathbf{v}_{2}$, changes its sign and is reduced by a factor $1-r$, with the coefficient of restitution $r$. Therefore, the kinetic energy related to the normal component is reduced by the factor $\epsilon^{2}=\frac{1-r^{2}}{2} \simeq 1-r$ for $r \approx 1$. The elastic limit, $r=1$, implies no dissipation $(\epsilon=0)$, while $r<1$ implies $\epsilon>0$.
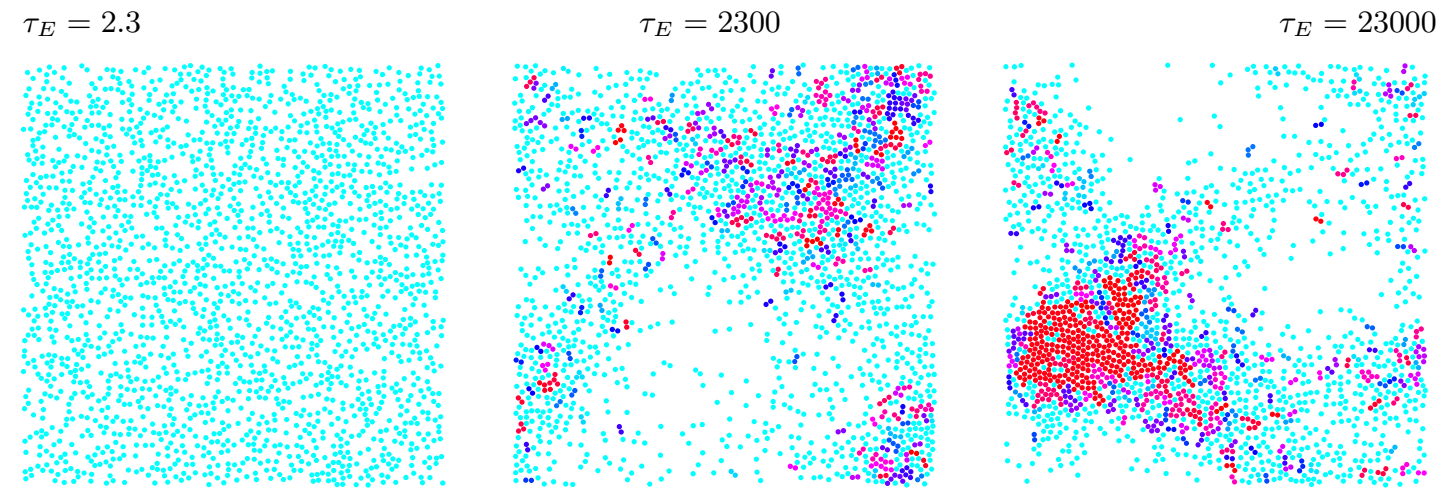

Fig. 1. (Color online) Two dimensional ED simulation snapshots at different dimensionless times $\tau_{E}$, for a periodic system with $N=2037$ particles, volume fraction $\nu=0.25$, and a coefficient of restitution $r=0.9$. The color indicates if the particle is part of a cluster defined by distance, relative velocity, and angle of movement, as explained in the main text.

\subsection{Free Cooling Granular Gas}

Three snapshots at different (dimensionless) times, $\tau_{E}=\hat{t} / \hat{t}_{E}(0)$, with (dimensional) time $\hat{t}$ and initial collision rate $\hat{t}_{E}^{-1}(0)$, as defined in Eq. (7), are displayed in Fig. 1. Different colors 
(turquoise/light-gray for single particles) correspond to particles forming different "clusters". Clusters are defined as particles that are closer than $0.1 d$ and their relative angle of movement, given by $\arccos \left(\mathbf{v}_{1} \cdot \mathbf{v}_{2} /\left(\left|\mathbf{v}_{1}\right|\left|\mathbf{v}_{2}\right|\right)\right)$, is smaller than $30^{\circ}$. This definition is similar to the one used in Ref. [20], but differs from previous work [6,17], however, the analysis of these differences goes beyond the scope of this paper.

\section{Hydrodynamics}

Consider a monodisperse system of particles of fixed mass whose collisions conserve momentum. The conservation of total mass and momentum is the (standard) basis for the macroscopic balance equations presented below. While mass and momentum are conserved, energy can be dissipated.

The constitutive relations needed to close the balance equations can be obtained on the basis of certain models and assumptions [3,21,22], part of which are presented below. In this section, dimensional quantities are indicated by a hat.

\subsection{Mass balance}

Assume that $N$ particles with total mass, $\hat{M}=\sum_{p \in V} \hat{m}$, reside in a certain representative volume element (RVE), with volume $\hat{V}$. The continuity equation for the density $(\hat{\rho}=\hat{M} / \hat{V})$ reads:

$$
\frac{D \hat{\rho}}{D \hat{t}}+\hat{\rho} \frac{\partial \hat{u}_{i}}{\partial \hat{x}_{i}}=0
$$

with the average streaming velocity components, $\hat{u}_{i}=(1 / \hat{M}) \sum_{p \in V} \hat{m} \hat{v}_{i}^{p}$, the particle mass, $\hat{m}$, and the particle velocity components, $\hat{v}_{i}^{p}$. The substantial derivative (or material derivative) is defined as: $\frac{D}{D \hat{t}}=\frac{\partial}{\partial \hat{t}}+\hat{u}_{i} \frac{\partial}{\partial \hat{x}_{i}}$, and the sum over equal indices is implied.

\subsection{Momentum balance}

Momentum-conservation allows the momentum density $\hat{\rho} \hat{u}_{i}$ to change with time, not only due to a (momentum carrying) flux, $\hat{\rho} \hat{u}_{i} \hat{u}_{k}$, in or out of the RVE, but also due to inhomogeneous/directed forces (like during collisions) exerted from the outside on its interior, leading to:

$$
\hat{\rho} \frac{D \hat{u}_{i}}{D \hat{t}}=-\frac{\partial \hat{\sigma}_{i j}}{\partial \hat{x}_{j}},
$$

with the stress tensor components $\hat{\sigma}_{i j}$ on the right hand side. The stress can be split into an isotropic and a deviatoric part, $\hat{\sigma}_{i j}=\hat{p}^{I} 1_{i j}+\hat{\sigma}_{i j}^{D}$, with (isotropic) pressure, $\hat{p}^{I}$, where $1_{i j}$ denotes the unit tensor.

For a Newtonian fluid, the deviatoric stress is proportional to the rate of shear strain (symmetric, trace-free velocity gradient):

$$
\hat{\sigma}_{i j}^{D}=-2 \hat{\mu}\left(\frac{1}{2}\left[\frac{\partial \hat{u}_{i}}{\partial \hat{x}_{j}}+\frac{\partial \hat{u}_{j}}{\partial \hat{x}_{i}}\right]-\frac{1}{\mathcal{D}} \frac{\partial \hat{u}_{k}}{\partial \hat{x}_{k}} 1_{i j}\right),
$$

with the shear viscosity $\hat{\mu}$. Note that the isotropic pressure, $\hat{p}^{I}=\hat{p}+\hat{p}_{\zeta}$, also contains viscous terms, proportional to volume changes (divergence of the velocity field $\partial \hat{u}_{k} / \partial \hat{x}_{k}$ ), which are explicitly subtracted in Eq. (4), for $\mathcal{D}=2,3$ dimensions. The isotropic, strain rate dependent stress, $\hat{p}_{\zeta}=-\hat{\zeta} \frac{\partial \hat{u}_{k}}{\partial \hat{x}_{k}}$, contains the bulk viscosity, $\hat{\zeta}$. The pressure will be assumed to be dominated by its rate independent part, unless otherwise mentioned. 


\subsection{Energy balance}

Energy-balance involves the kinetic energy density, $\hat{\rho} \hat{u}^{2} / 2$, due to the streaming velocity, $\hat{u}_{i}$, and $\hat{u}^{2}=\hat{u}_{i} \hat{u}_{i}$. In addition, there is also a fluctuating energy density, related to the fluctuating velocity $\hat{v}_{T}$ and to the "granular temperature",

$$
\hat{T}_{g}=\frac{2 \hat{E}_{\mathrm{kin}}}{\mathcal{D} N}=\frac{1}{\mathcal{D} N} \sum_{p \in V} \hat{m}^{p}\left(\hat{v}_{i}^{p}-\hat{u}_{i}\right)^{2}=\frac{\hat{m} \hat{v}_{T}^{2}}{\mathcal{D}},
$$

i.e., twice the fluctuating kinetic energy per particle per degree of freedom, where the sum runs over all $N$ particles in the averaging volume $V$. Note that in the following, we use $\hat{T}=\hat{T}_{g} / \hat{m}=$ $\hat{v}_{T}^{2} / 2$. The energy-density balance then reads:

$$
\hat{\rho} \frac{D}{D \hat{t}} \hat{T}=-\hat{\sigma}_{i k} \frac{\partial \hat{u}_{i}}{\partial \hat{x}_{k}}-\frac{\partial \hat{q}_{k}}{\partial \hat{x}_{k}}-\hat{\gamma} \hat{T}
$$

with the energy density dissipation rate, $-\hat{\gamma} \hat{T}$. The right hand side of Eq. (5) contains also the rate of shear-heating, and the divergence of the heat-flux. The latter contains the classical term proportional to the temperature gradient with the thermal conductivity, $\hat{\kappa}$, and a second, nonclassical term, proportional to the density gradient with the corresponding transport coefficient $\hat{\lambda}[7,21]$, so that:

$$
\hat{q}_{k}=-\hat{\kappa} \frac{\partial \hat{T}}{\partial \hat{x}_{k}}-\hat{\lambda} \frac{\partial \hat{\rho}}{\partial \hat{x}_{k}} .
$$

Below we neglect the "non-classical" term since it is proportional to $1-r^{2}$ and we specialize to near-elastic systems. The validity of this assumption has to be studied in future works.

\subsection{The ("classical") transport coefficients}

The equation of state (for the pressure), expressions for the shear and bulk viscosities and the heat conductivity, as well as the expression for the dissipation rate of the energy density, are collectively referred to as "expressions for the transport coefficients", for the sake of brevity. They are, a priori, not constant but dependent on the hydrodynamic fields and, possibly, also on their gradients or other terms, which are not considered and discussed here.

\subsubsection{Transport coefficients in 2D}

In $2 \mathrm{D}$, for a single species, in the elastic limit, $r \rightarrow 1$, in lowest order in powers of $1-r^{2}$ and the gradients, the transport coefficients can be expressed in various forms, see Ref. [22] for a detailed review.

All transport coefficients are proportional to $\hat{\rho}=\hat{\rho}^{p} \nu$, to powers of $\hat{v}_{T}$, and to powers of the product $s=s_{*} / \nu=(\nu g(\nu))^{-1}$, i.e., proportional to the non-dimensional mean free path, which depends on the pair-correlation function $g(\nu)$. If one divides the transport coefficients by the combination $\hat{\rho} \hat{t}_{E}^{-1}$ that is common to all (see Luding's notation [22]), only powers of $\hat{d}, \hat{v}_{T}$ and $s$ remain as variables:

$$
\begin{aligned}
& \hat{p}=\hat{\rho} \hat{t}_{E}^{-1} \hat{v}_{T} \frac{\hat{d}}{2 \sqrt{2} \tilde{\gamma}}(s+2), \\
& \hat{\mu}=\hat{\rho} \hat{t}_{E}^{-1} \frac{\hat{d}^{2}}{4 \tilde{\gamma}^{2}}\left(s^{2}+2 s+\left(1+\frac{8}{\pi}\right)\right), \\
& \hat{\zeta}=\hat{\rho} \hat{t}_{E}^{-1} \frac{\hat{d}^{2}}{4}
\end{aligned}
$$




$$
\begin{aligned}
\hat{\kappa} & =\hat{\rho} \hat{t}_{E}^{-1} \frac{\hat{d}^{2}}{\tilde{\gamma}^{2}}\left(s^{2}+3 s+\left(\frac{9}{4}+\frac{4}{\pi}\right)\right), \\
\hat{\gamma} \hat{T} & =\hat{\rho} \hat{t}_{E}^{-1} \epsilon^{2} \frac{\hat{v}_{T}^{2}}{2},
\end{aligned}
$$

with $\tilde{\gamma}=4 / \sqrt{\pi}$, and hiding the proportionality

$$
\hat{\rho} \hat{t}_{E}^{-1}=\sqrt{2} \tilde{\gamma} \frac{\hat{\rho} \hat{v}_{T}}{\hat{d} s} \propto \frac{\nu \hat{v}_{T}}{s}
$$

with the collision rate $\hat{t}_{E}^{-1}=2 \tilde{\gamma} \sqrt{\hat{T}} /(\hat{d} s)$. These equations have been obtained from a ChapmanEnskog expansion applied to the Enskog-Boltzmann equation in Ref. [23] and are frequently cited, e.g. in Refs. [10,24-26].

In order to compare our results with the ones shown in Ref. [3], we normalize and nondimensionalize the hydrodynamic equations. Lengths are scaled with particle diameter $\hat{d}$, masses by particles mass $\hat{m}$, and velocities with the initial thermal velocity $\sqrt{\hat{T}_{0}}=\hat{v}_{T}(0) / \sqrt{2}$. The hat that denotes the dimensional nature of the variable will be dropped after non-dimensionalization, as for example:

$$
\hat{u}_{i}=\hat{T}_{0}^{1 / 2} u_{i}, \hat{T}=\hat{T}_{0} T, \hat{x}=\hat{d} x, \hat{t}=\hat{d} \hat{T}_{0}^{-1 / 2} t .
$$

Consequently, the transport coefficients, in McNamara's notation [3], are defined as:

$$
\begin{aligned}
\hat{p} & =\tilde{p}(\nu)\left[\hat{\rho}^{p} \hat{d} \frac{\hat{T}}{\hat{s}}\right], \\
\hat{\mu} & =\tilde{\mu}(\nu) \nu^{-1}\left[\hat{\rho}^{p} \hat{d}^{2} \frac{\hat{T}^{1 / 2}}{\hat{s}}\right], \\
\hat{\zeta} & =\tilde{\zeta} \nu\left[\hat{\rho}^{p} \hat{d}^{2} \frac{\hat{T}^{1 / 2}}{\hat{s}}\right], \\
\hat{\kappa} & =\tilde{\kappa}(\nu) \nu^{-1}\left[\hat{\rho}^{p} \hat{d}^{2} \frac{\hat{T}^{1 / 2}}{\hat{s}}\right], \\
\hat{\gamma} \hat{T} & =2 \epsilon^{2} \tilde{\gamma} \nu\left[\hat{\rho}^{p} \frac{\hat{T}^{3 / 2}}{\hat{s}}\right],
\end{aligned}
$$

with $\hat{s} \equiv s \hat{d}$. The positive non-dimensional functions $\tilde{p}(\nu), \tilde{\mu}(\nu), \tilde{\zeta}, \tilde{\kappa}(\nu)$, and $\tilde{\gamma}$ are of order $\mathcal{O}(1)$ for any value of $\nu$. Their explicit form, as used in the next section, is given by:

$$
\begin{aligned}
\tilde{p}(\nu) & =s_{*}+2 \nu, \\
\tilde{\mu}(\nu) & =\frac{\sqrt{\pi}}{8}\left(s_{*}+\nu\right)^{2}+\frac{\nu^{2}}{\sqrt{\pi}}, \\
\tilde{\zeta} & =\frac{2}{\sqrt{\pi}}, \\
\tilde{\kappa}(\nu) & =\frac{\sqrt{\pi}}{2}\left(s_{*}+\frac{3 \nu}{2}\right)^{2}+\frac{2 \nu^{2}}{\sqrt{\pi}}, \\
\tilde{\gamma} & =\frac{4}{\sqrt{\pi}} .
\end{aligned}
$$

Note that the last term in the heat conductivity is different by a factor $2 \nu^{2}$ from McNamara [3], what we think is due to a typo in his paper, however, this term has a small effect only. 


\subsubsection{Pair-correlation functions}

Note that the pair correlation function $g(\nu)$ has not been specified as yet. In this paper we will consider, as a working example, the differences between four of them: the classical (Enskog) function proposed by Henderson [27]:

$$
g_{2}(\nu)=\frac{1-7 \nu / 16}{(1-\nu)^{2}}
$$

the one proposed by Grossman et al. in Ref. [28],

$$
g_{\mathrm{pm}}(\nu)=1 /\left(\nu_{m}-\nu\right),
$$

where $\nu_{m}=\pi /(2 \sqrt{3})$ is the closest packing fraction in $2 \mathrm{D}$, and the high density limit of the previous one

$$
g_{\mathrm{fv}}(\nu)=g_{\mathrm{pm}}+(2 \nu)^{-1},
$$

which is evidently wrong at low densities [22,29], and finally $g_{Q}(\nu)$, an empirical mix between $g_{2}$ and $g_{\mathrm{fv}}$, which takes into account the disorder-order phase transition at $\nu=0.7$, for which the explicit definition can be found in Ref. [22].

These functions are plotted in Fig. 2 to illustrate the differences. When compared with the non dimensional pressure obtained from ED simulations [29], the classical formula does not have the correct behavior for high densities. In the range $\nu>0.75$, the high density limit formula $g_{\mathrm{fv}}$ behaves well, while $g_{\mathrm{pm}}$ behaves qualitatively well for all densities. The empirical $g_{Q}(\nu)$ performs quantitatively correct at all densities, including the disorder-order phase transition at $\nu_{c} \simeq 0.699$. In the inset of Fig. 2 we plot $P=2 \nu g(\nu)$, i.e., the non-dimensional collisional pressure. As can be seen from the data, when zooming in closer [29], the slope for $2 \nu g_{Q}(\nu)$ is always positive, whereas $g(\nu)$ has a negative slope in the transition zone.

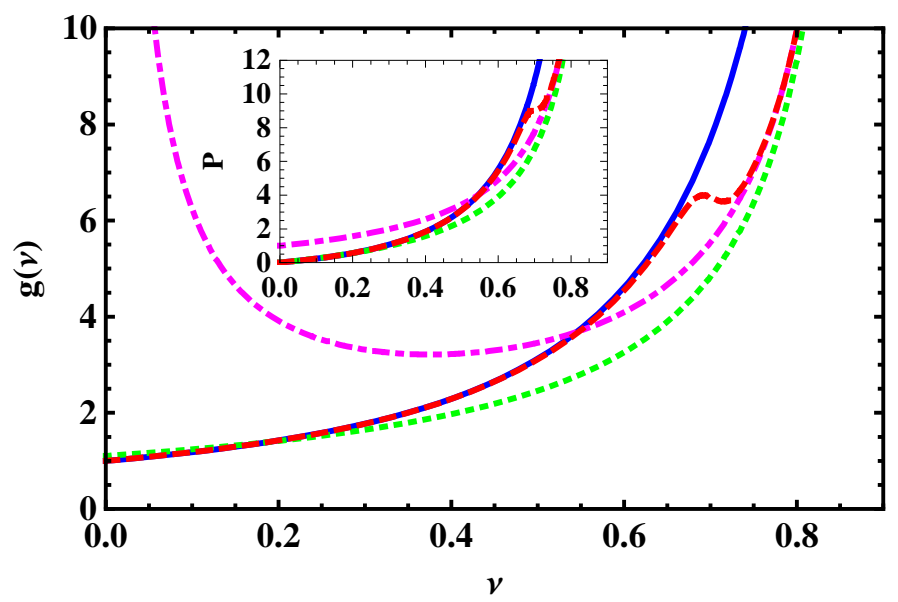

Fig. 2. (Color online) Pair-correlation function at contact. The solid blue line corresponds to $g_{2}$, the magenta dot-dashed line to $g_{\mathrm{fv}}$, the green dotted line to $g_{\mathrm{pm}}$ and the red dashed line to $g_{Q}$. The slope of the $g_{Q}$ curve around $\nu_{c}$ is strongly determined by the numerical coefficients, the width of the transition between low and high densities is $m_{c}=0.0111$, and the critical density is $\nu_{c}=0.699$, as in Ref. [22]. In the inset, the non-dimensional collisional pressure, $P=2 \nu g(\nu)$ is plotted against $\nu$.

In the rest of the paper, we will assume that Eqs. 2), (3), and (5) are complete and sufficient to describe arbitrary flow conditions and rheology. This ansatz implies, that the flow behavior of very dense, realistic granular matter can be already rather well described, in most cases, by using the constitutive relations in Eqs. (6) with an improved version of the pair-correlation function $g(\nu)$. In the following we will study how this works in the case of the stability analysis of the homogeneous cooling state. 


\section{Stability Analysis}

The homogeneous reference solution for a freely cooling gas is found taking all the spatial derivatives in the hydrodynamic equations equal to zero, leaving just equation (5), non-dimensionalized as described above (and dropping density that occurs on both sides):

$$
\frac{d T}{d t}=-\frac{2 \tilde{\gamma} \epsilon^{2}}{s_{0}} T^{3 / 2}
$$

The solution is known as Haff's law [1], and can be written (following the notation of Ref. [3]):

$$
T(t)=\frac{1}{\left(\epsilon^{2} \tilde{\gamma} t / s_{0}+1\right)^{2}},
$$

with the other fields remaining homogeneous and constant:

$$
\nu=\nu_{0}, u:=u_{x}=0, v:=u_{y}=0 .
$$

The homogeneous-state variables and coefficients are designated by a subscript " 0 " $\left(\nu_{0}, p_{0} \equiv\right.$ $p\left(\nu_{0}\right), s_{0} \equiv s\left(\nu_{0}\right)$, etc.), and the derivatives with respect to density (evaluated at $\left.\nu_{0}\right)$ are denoted as, e.g., $p_{\nu_{0}} \equiv d p /\left.d \nu\right|_{\nu_{0}}$.

\subsection{Collision rate}

Starting from the initial configuration with homogeneous $\nu_{0}$ and $T_{0}$, the system dissipates energy at a rate that also depends on the energy of the system. As the energy of the system is dissipated, the relative velocities of the particles become smaller. The dynamics becomes slower, and with this, less energy is dissipated in each collision. The natural time unit of the system is thus the number of collisions per particle, given by the integral of the non-dimensional collision frequency $f(t):=T(t)^{1 / 2}=t_{E}^{-1}(t) \hat{d} s /\left(2 \tilde{\gamma} \hat{T}_{0}^{1 / 2}\right)$ :

$$
\tau \equiv \frac{1}{s_{0}} \int_{0}^{t} f\left(t^{\prime}\right) d t^{\prime}=\frac{1}{\epsilon^{2} \tilde{\gamma}} \log \left(1+\frac{\epsilon^{2} \tilde{\gamma} t}{s_{0}}\right)=-\frac{\log (f(t))}{\epsilon^{2} \tilde{\gamma}} .
$$

\subsection{Linear equations}

In the following, we study the linear stability of the system in its new time variable, $\tau$, since then the equations can be written in the form $d \mathbf{Z}^{\prime} / d \tau=F\left(\mathbf{Z}^{\prime}\right)$, with the vector of perturbed fields $\mathbf{Z}^{\prime}$. If we would keep the physical time as our variable, the equation for the temperature would be explicitly time-dependent.

In order to linearize the hydrodynamic equations about the homogeneous but time-dependent solution (9), the fields are split into their homogeneous and their perturbation parts:

$$
\begin{aligned}
\nu & =\nu_{0}\left(1+\nu^{\prime}\right), \\
u & =f u^{\prime}, \\
v & =f v^{\prime}, \\
T & =f^{2}\left(1+T^{\prime}\right),
\end{aligned}
$$

where $\nu$ is the volume fraction, $u(v)$ the bulk velocity in $x(y)$ direction, and the primes indicate the perturbations. The primed quantities define $\mathbf{Z}^{\prime}$, and the factor $f$ is introduced for later convenience.

The final goal of this section is to examine the linear stability of small perturbations of the form $\exp (i \mathbf{k} \cdot \mathbf{x})$. Using Squire's theorem, that is, projecting the $2 D$ problem into one dimension, we will consider perturbations only along the $x$ direction so that the derivatives in 
the $y$ direction vanish. Taking the perturbations up to first order gives four coupled equations, by inserting the fields into Eqs. (2), (3) and (5):

$$
\begin{aligned}
\nu_{t}^{\prime} & =-f u_{x}^{\prime}, \\
\nu_{0}\left(f u^{\prime}\right)_{t} & =-p_{x}^{\prime}+\left(\mu_{0}+\zeta_{0}\right) f u_{x x}^{\prime}, \\
\nu_{0}\left(f v^{\prime}\right)_{t} & =\mu_{0} v_{x x}^{\prime} \\
\nu_{0}\left(f^{2} T^{\prime}\right)_{t} & =-p_{0} f u_{x}^{\prime}+\kappa_{0} f^{2} T_{x x}^{\prime}-\gamma_{0} f^{2} T^{\prime}-\gamma^{\prime} f^{2}-2 \nu_{0} f f_{t} \nu^{\prime},
\end{aligned}
$$

where the explicit expressions for the non-dimensional transport coefficients in the homogeneous state, $p_{0}, \mu_{0}, \zeta_{0}, \gamma_{0}$, and their perturbed values $p^{\prime}$ and $\gamma^{\prime}$ can be found in Ref. [3]

Changing to the new time variable $\tau$, using the identities $f=\exp \left(-\epsilon^{2} \tilde{\gamma} \tau\right), d \tau / d t=f / s_{0}$, and scaling lengths by density as $X=\nu_{0} x$ and thus wave numbers as $K=\nu_{0}^{-1} k$, gives [3]:

$$
\begin{aligned}
& \nu_{\tau}^{\prime}=-s_{0}^{*} u_{X}^{\prime}, \\
& u_{\tau}^{\prime}=-\tilde{p}_{0}\left(T_{X}^{\prime}-s_{X}^{\prime}\right)-\tilde{p}_{\nu_{0}} \nu_{0} \nu_{X}^{\prime}+\left(\tilde{\mu}_{0}+\nu_{0}^{2} \tilde{\zeta}\right) u_{X X}^{\prime}+\epsilon^{2} \tilde{\gamma} u^{\prime}, \\
& v_{\tau}^{\prime}=\tilde{\mu}_{0} v_{X X}^{\prime}+\epsilon^{2} \tilde{\gamma} v^{\prime}, \\
& T_{\tau}^{\prime}=-\tilde{p}_{0} u_{X}^{\prime}+\tilde{\kappa}_{0} T_{X X}^{\prime}+2 \epsilon^{2} \tilde{\gamma} s^{\prime}-\epsilon^{2} \tilde{\gamma} T^{\prime} .
\end{aligned}
$$

Because the coefficients do not explicitly depend on time, we can perform the stability analysis for any choice of the pair correlation function $g(\nu)$, as long as it does not explicitly depends on temperature.

\subsection{Shear instability}

The equation for the transverse velocity perturbation, $v^{\prime}$, that is the shear mode, is decoupled from the others. Assuming $v^{\prime}=\breve{v} \exp (\sigma \tau+i K X)$, with $\sigma$ the grow rate and the checked quantity, $\check{v}$, a constant, Eq. (17) reads:

$$
\sigma=-\tilde{\mu}_{0} K^{2}+\epsilon^{2} \tilde{\gamma}
$$

indicating growing shear instabilities for perturbations with wave numbers $K<K_{c}^{\text {shear }} \equiv$ $\epsilon\left(\tilde{\gamma} / \tilde{\mu}_{0}\right)^{1 / 2}$.

In Fig. 3 we present the critical shear mode wave numbers (solid blue lines) as functions of the density for the four pair-correlation functions. Just the $g_{\mathrm{fv}}$ solution behaves differently (and incorrectly) for low densities, while the other three functions yield similar results since the dependence of $\tilde{\mu}_{0}$ on the pair-correlation function goes as $g(\nu)^{-2}$. In order to quantify the difference between the classical value and the one obtained with the modified pair correlation function, we plot the shear mode onset criterion normalized by the classical prediction (in Fig. 4 left). As expected, the only differences between $g_{2}$ and $g_{Q}$ occur for $\nu>0.65$, nevertheless, they are very small $(\sim 2 \%)$. In the case of $g_{\mathrm{pm}}$, for low densities it predicts an up to $10 \%$ bigger critical wavelength than $g_{2}$ ( since $g_{\mathrm{pm}}(\nu \sim 0)>g_{2}(\nu \sim 0)$ ), while for intermediate densities it predicts an up to $5 \%$ smaller one. For high densities the prediction of $g_{\mathrm{pm}}, g_{\mathrm{fv}}$ and $g_{Q}$ are practically identical.

\subsection{Heat and sound instabilities}

Now, we must resolve three remaining coupled equations with five variables, which will give us the dispersion relation $\sigma=\sigma(K)$. Since $s=s(\nu)$ one has $s^{\prime}=\left.\frac{\nu_{0}^{2}}{s_{0}^{*}}\left(\frac{d s}{d \nu}\right)\right|_{\nu_{0}} \nu^{\prime}$, with this, and using the same plane wave ansatz as before, the equations read:

$$
\begin{aligned}
\sigma \check{\nu} & =-s_{0}^{*} i K \check{u} \\
\sigma \check{u} & =-\tilde{p}_{0}(i K \check{T}-i K \check{s})-i K \tilde{p}_{\nu_{0}} \nu_{0} \check{\nu}-K^{2}\left(\tilde{\mu}_{0}+\nu_{0}^{2} \tilde{\zeta}\right) \check{u}+\epsilon^{2} \tilde{\gamma} \check{u} \\
\sigma \check{T} & =-i K \tilde{p}_{0} \check{u}-K^{2} \tilde{\kappa}_{0} \check{T}+2 \epsilon^{2} \tilde{\gamma} \check{s}-\epsilon^{2} \tilde{\gamma} \check{T}
\end{aligned}
$$



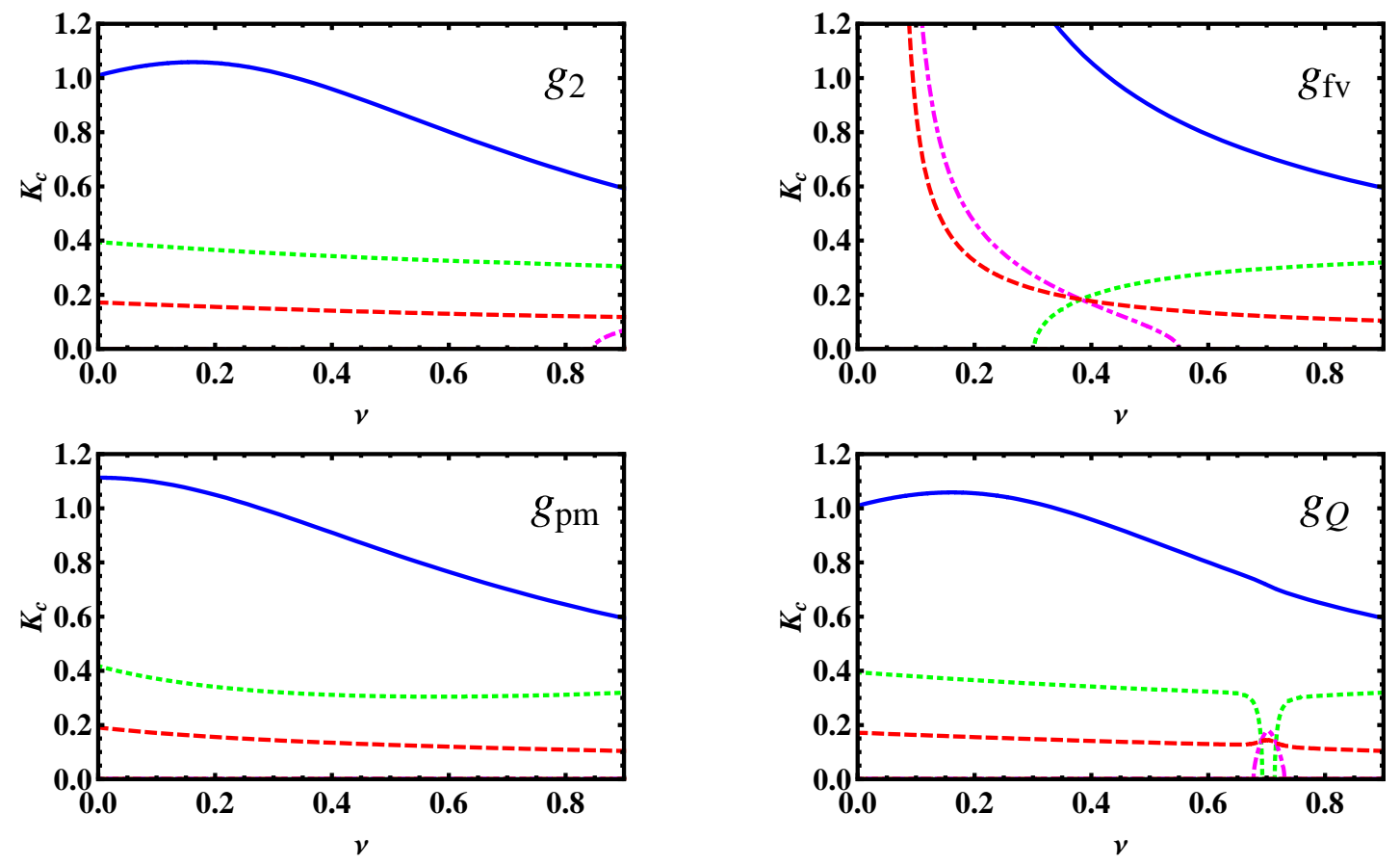

Fig. 3. (Color online) The critical wave numbers $K_{c}^{\text {shear }}$ (solid-blue) and $K_{c}^{\text {heat }}$ (dotted-green), together with the other stability criteria (see [3]): $b>0$ (dashed-red) and $a b-c>0$ (dash-dotted-magenta). In the upper left figure we used $g_{2}$, in the upper right $g_{\mathrm{fv}}$, in the bottom left we used $g_{\mathrm{pm}}$, and in the bottom right $g_{Q}$.

From Eq. (15) we have $\check{\nu}=-\frac{i K s_{0}^{*}}{\sigma} \check{u}$. Substituting the values of $\check{\nu}$ and $\check{s}$, and defining $a^{*} \equiv-\left.\nu_{0}^{2}\left(\frac{d s}{d \nu}\right)\right|_{\nu_{0}}$, we can write $\check{s}=i K a^{*} \check{u} / \sigma$, the dispersion relation is found to be:

$$
\begin{array}{r}
\sigma\left(\sigma+K^{2} \tilde{p}_{0} \sigma a^{*}+\tilde{p}_{\nu_{0}} \nu_{0} K^{2} \frac{s_{0}^{*}}{\sigma}+K^{2}\left(\tilde{\mu}_{0}+\nu_{0}^{2} \tilde{\zeta}\right)-\epsilon^{2} \tilde{\gamma}\right)\left(\sigma+\tilde{\kappa}_{0} K^{2}+\epsilon^{2} \tilde{\gamma}\right)+ \\
K^{2} \tilde{p}_{0}^{2} \sigma-2 \epsilon^{2} \tilde{\gamma} a^{*} K^{2} p_{0}=0 .
\end{array}
$$

This equation can be written in the form $\sigma^{3}+a \sigma^{2}+b \sigma+c=0$. It can be shown [3] that if any of $a, b, c$ or $a b-c$ are negative, then there exists a $\sigma$ with a positive real part, i.e., there exists an unstable mode. In Eq. (19), $a$ is always positive, but $b, c$ and $a b-c$ can become negative, indicating a growing instability.

The above criteria, together with the critical wavelength $K_{c}^{\text {shear }}$ are shown in Fig. 3 for different pair-correlation functions and a fixed coefficient of restitution $r=0.9$. Qualitatively $g_{2}, g_{\mathrm{pm}}$ and $g_{Q}$ have the same shape. Since $g_{\mathrm{fv}}$ is incorrect for low densities (and will not be further analyzed), the predictions are not valid and it even adds a new mechanism of instability for densities below $\nu \sim 0$.4. Since $g_{\mathrm{pm}}(\nu \sim 0) \neq g_{2}(\nu \sim 0)$, their critical wavelengths differ somewhat $(10 \%)$ for low densities.

Like for the shear mode, the predictions for the heat mode associated to $g_{2}$ and $g_{Q}$ are almost equal for low and moderate densities, differing around the critical density $\nu_{c} \approx 0.7$ and above. In the transition region, the theory based on $g_{Q}$ predicts a critical wavelength that is about $40 \%$ smaller than the classical value, and a new unstable mode appears.

In Fig. 5, we show the dispersion relation of the unstable modes, i.e. the positive roots of Eq. (19), for $g_{2}$ and $g_{Q}$ as a function of the scaled wave number $K=\nu_{0}^{-1} k$. The density is increasing from the top- to the bottom-line, and the coefficient of restitution is again $r=0.9$. The dispersion relation for $g_{\mathrm{pm}}$ (away from $\nu=0.7$ ) is qualitatively similar to the one predicted by $g_{Q}$ and therefore is not shown. 

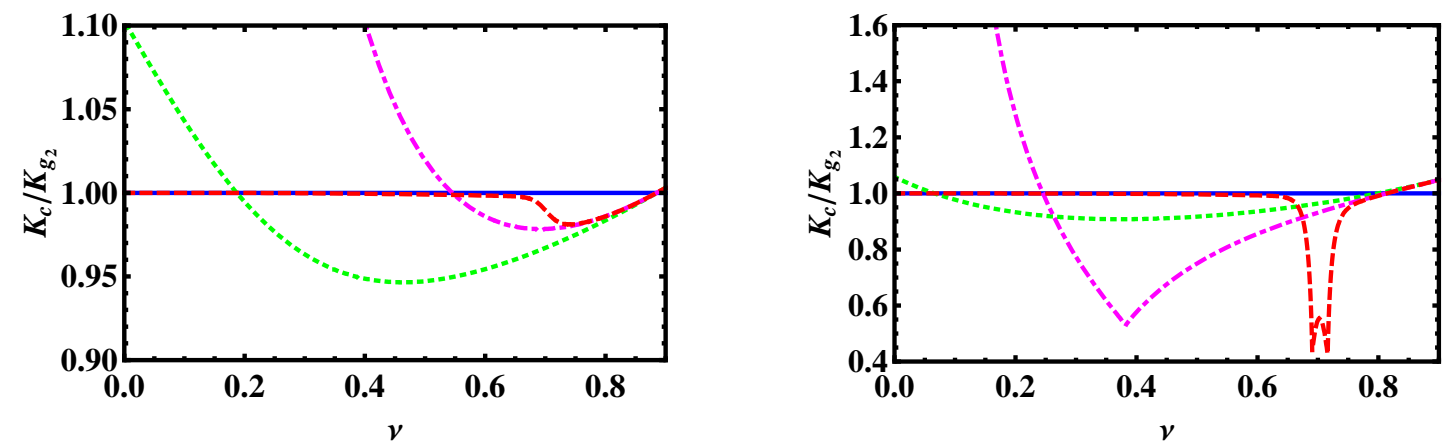

Fig. 4. (Color online) The critical wave numbers $K_{c}^{\text {shear }}$ (left) and $K_{c}^{\text {heat }}$ (right) for the four different pair-correlation functions normalized by the prediction given by the classical pair-correlation function $g_{2}$, and a fixed coefficient of restitution $r=0.9$. The solid blue line indicates identity and corresponds to $g_{2}$, the magenta dot-dashed line corresponds to $g_{\mathrm{fv}}$, the green dotted line corresponds to $g_{\mathrm{pm}}$ and the red dashed line corresponds to $g_{Q}$. For the shear mode the variations are of the order of $10 \%$ while for the heat mode the variations can be as big as $40 \%$ in the case of $g_{Q}$ (note the different scale in the vertical axis for both plots).
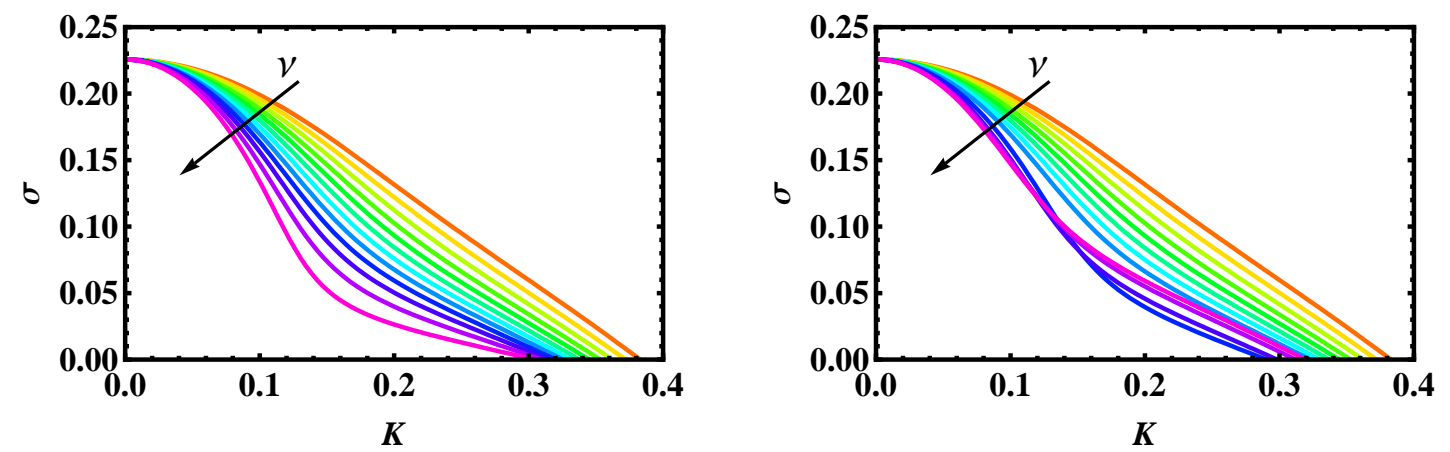

Fig. 5. (Color online) The dispersion relation for different $\nu$ increasing from $\nu=\Delta \nu=0.075$ up to $\nu=0.9$ in steps of $\Delta \nu$. In the upper left figure we used $g_{2}$, in the right $g_{Q}$, with a coefficient of restitution $r=0.9$.

For $\nu \geq 0.75$ the critical wave number $K_{c}$ (where the lines cross the horizontal axis) changes behavior when $g_{Q}$ is used, i.e., the critical wave number is smaller (relative to the one predicted by $\left.g_{2}\right)$ at densities $0.675 \leq \nu \leq 0.85$. Also, the shape of the dispersion relation is different. For the highest density, $\nu=0.9$, the critical $K_{c}$ is increased due to excluded volume effects, relative to the classical case with $g_{2}$.

For densities around $\nu=0.7$ a new mode appears for $g_{Q}$. As seen in Fig. 6 , for $\nu=0.7$ and $r=0.9$, the dispersion relation has two positive roots that merge around $K=0.115$. To illustrate the differences between the two new modes and the classical one, we plot the eigenmodes for $K=0.1$ in Fig. 7. The classical mode (Fig. 7 left) has $\sigma=0.158$ and is a temperature mode with small amplitude for velocity and density, which are shifted by $\pm \pi / 2$ respectively, from the temperature. The first new mode (Fig. 7 center) has $\sigma_{1}=0.135$, and is mainly a temperature mode, with small amplitude for velocity and a yet smaller amplitude for density, which are in phase and shifted by $-\pi / 2$ from the temperature. This mode is analogous to the classical mode (Fig. 7 left) but the velocity is shifted by $-\pi / 2$. The second new mode (Fig. 7 right) has $\sigma_{2}=0.020$ and the three fields have comparable amplitudes. The temperature is again the biggest mode, followed by the density and then the velocity. The phase shift is the same as in the previous mode, that is, velocity and density are shifted by $-\pi / 2$ from the temperature. 

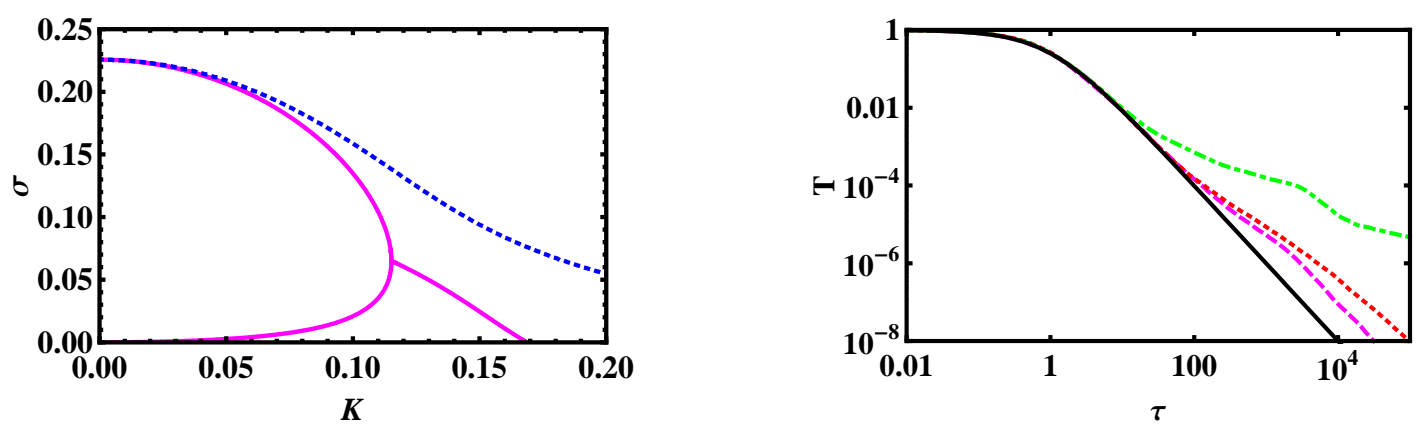

Fig. 6. (Color online) (Left) Dispersion relation for $\nu=0.7$ and $r=0.9$ for different pair-correlation functions. The magenta solid line is the one obtained by using $g_{Q}$, while the classical one is the dotted blue line. The onset of the instability is shifted to smaller $K$ when using $g_{Q}$. The bifurcation starts at $K_{b} \simeq 0.115$ and the new mode appears. (Right) The non-dimensional temperature as a function of the non-dimensional time, for $L=20$ magenta dashed line, $L=30$ red dotted line, and $L=100$ green dot-dashed line.
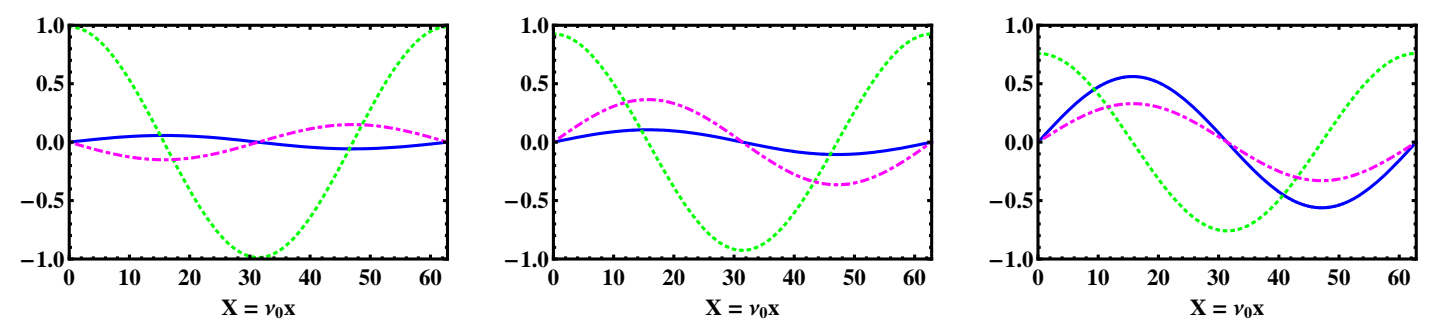

Fig. 7. (Color online) The unstable modes for $\nu=0.7, r=0.9, K=0.1$ using $g_{2}$ and $g_{Q}$. In the left panel, the modes for the classical pair-correlation function, in the middle panel the $\sigma_{1}$ mode (the fast one), and in the right panel the $\sigma_{2}$ mode (the slow one) for $g_{Q}$ are shown. The solid blue line is the density, $\nu$, the dotted green line is the temperature, $T$, and the dot-dashed magenta line is the velocity in the $x$ direction, $u$.

\subsection{Numerical Simulations}

To test the predictions of the theory, numerical simulations were carried out for three systems with density $\nu=0.7$ and $r=0.9$, for different sizes $L=\hat{L} / \hat{d}=20,30$ and 100 . The minimal wave numbers are $K_{m}=2 \pi \nu \hat{d} / \hat{\lambda}_{c} \simeq 0.20,0.14$ and 0.04 , respectively (see Fig. 6), where $\hat{\lambda}_{c}$ is the dimensional wavelength of the marginally stable wave, i.e., the smallest size of the system above which the instability is expected.

The system is thermalized in a way similar to [2]: starting from a square lattice with uniform random velocities, the system evolves during at least 100 collisions per particle with $r=1$, so that a Maxwellian velocity distribution is reached. A small $\left(\delta u \sim 0.1 v_{T}\right)$ sinusoidal perturbation of the velocity (in $x$ direction) is added to the initial state of the system and its evolution with $r=0.9$ is studied. The temperature evolution for the three systems is presented in Fig. 6 (right). After initial agreement, the systems deviate from Haff's law (solid line), the largest system first, the smallest last, as expected from the dispersion relation (see Fig. 6 left).

The dependence of Haff's law on the pair-correlation function is through $s_{0}$. In Fig. 6 we have used $g_{Q}$, which fits the data better than $g_{2}$. The ratio between the two pair-correlation functions for this density, $q_{g}=g_{2}(0.7) / g_{Q}(0.7)$ is equal to the inverse of the ratio of the $s_{0}$, that is $s_{0}^{g_{2}} / s_{0}^{g_{Q}}=0.84=1 / q_{g}$.

To quantify the role of the clustering instability, we measure the power spectra for the density fluctuations and the horizontal velocity, on the $x$ axis, averaged over the $y$ direction in bins of size $d$. In Fig. 8 we present the power spectra at three different times $\tau \simeq 0.001,5$ and 1000 for the $L=100$ system, for two different initial conditions, one perturbed as described above and 
the original thermal configuration. The power spectra are normalized so that the relative power of each mode is displayed. This way we can perform comparisons between different times, for which the amplitude of the velocity fluctuations is decreasing. The only system that displays a clear peak for low wave numbers, for both density and velocity fluctuations, is the one with $L=100$. The system with $L=30$ presents a qualitatively similar behavior but slower, and the peaks are not as marked as for $L=100$. The $L=20$ system does not present a peak, showing that the clustering mode is not present and the deviation from Haff's law in this system is due to the shear mode instability.

In order to visualize the emergence of the clustering mode, a space-time diagram of the density fluctuations of the three systems is presented in Fig 9. The system with $L=20$ does not develop a sinusoidal profile, at least for the time scales studied here. In contrast, for $L=30$, it is possible to identify a sinusoidal perturbation at $\tau \sim 10^{4}$, while the system with $L=100$ presents a clear sinusoidal behavior already for $\tau \sim 10^{2}$, and later develops fluctuations with a larger wave number at $\tau \sim 10^{4}$.

The evolution of the smallest $K$, the one where the perturbation in the velocity is added, is presented in Fig. 10. The sinusoidal density mode that can be appreciated in Fig. 9, is reflected here in the growth of the relative power. For the system with $L=30$, a growth is observed at $\tau \sim 10^{2}-10^{4}$, while for $L=100$ the growth occurs earlier at $\tau \sim 0.1-10^{2}$. The smallest system does not present a clear growth of the field perturbation in the time scale studied.

To illustrate the change in density in real space, four snapshots of the perturbed system with $L=100$ are shown in Fig. 11. The first two snapshots from the left correspond to the same $\tau$ used in the right two plots of Fig. 8; the snapshot corresponding to $\tau=0.001$ is not shown due to its similarity with the one at $\tau=5$. The color code represents the kinetic energy of each particle normalized by the average temperature at that instant. The system develops density fluctuations (clustering) that grow with time, to finally arrive to a sheared regime with two shear bands, visible in the right panel of Fig. 11.
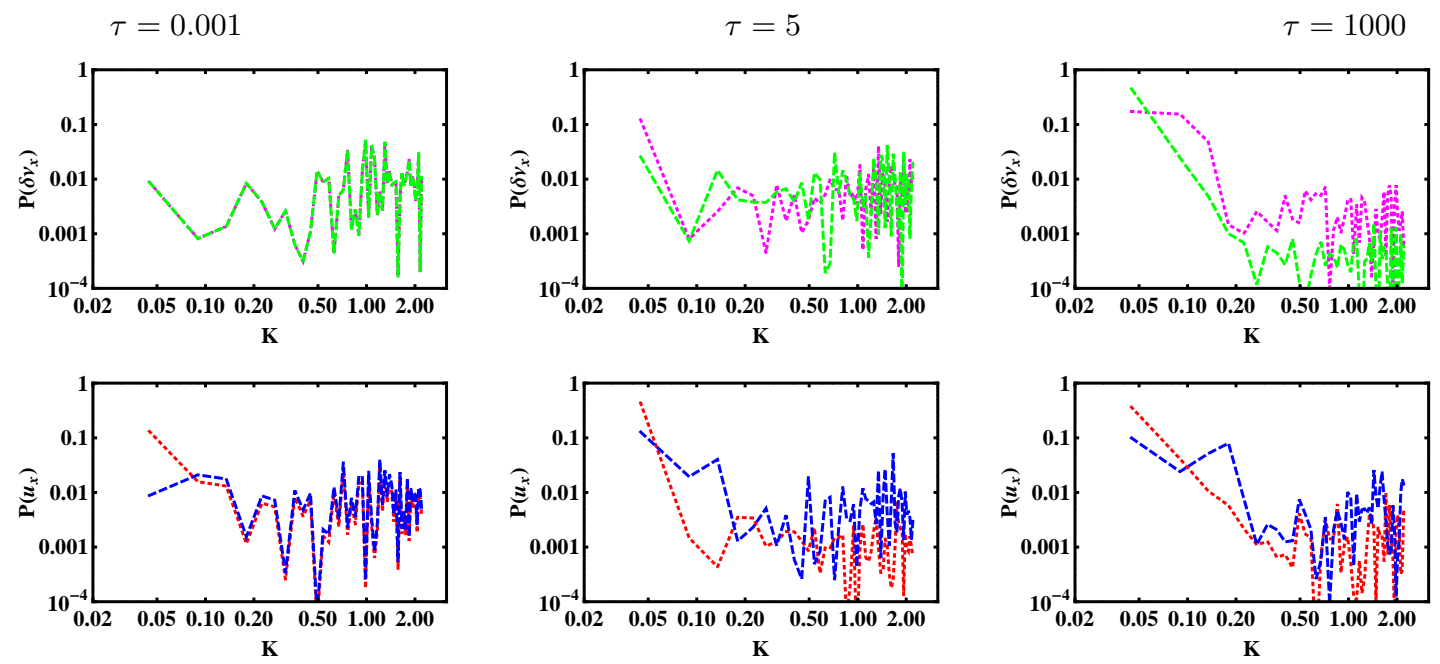

Fig. 8. (Color online) (Upper panel) Normalized power spectra of the density for three different times. The dotted magenta line corresponds to the perturbed system, and the dashed green line to the nonperturbed system. (Bottom panel) Normalized power spectra of the velocity in the $x$ direction, $u$, for the same three times as above. The red dotted line corresponds to the perturbed system, and the dashed blue line to the non-perturbed system. The contribution of the initial perturbation can be clearly seen in the first graph with the peak at $K \simeq 0.04$. 

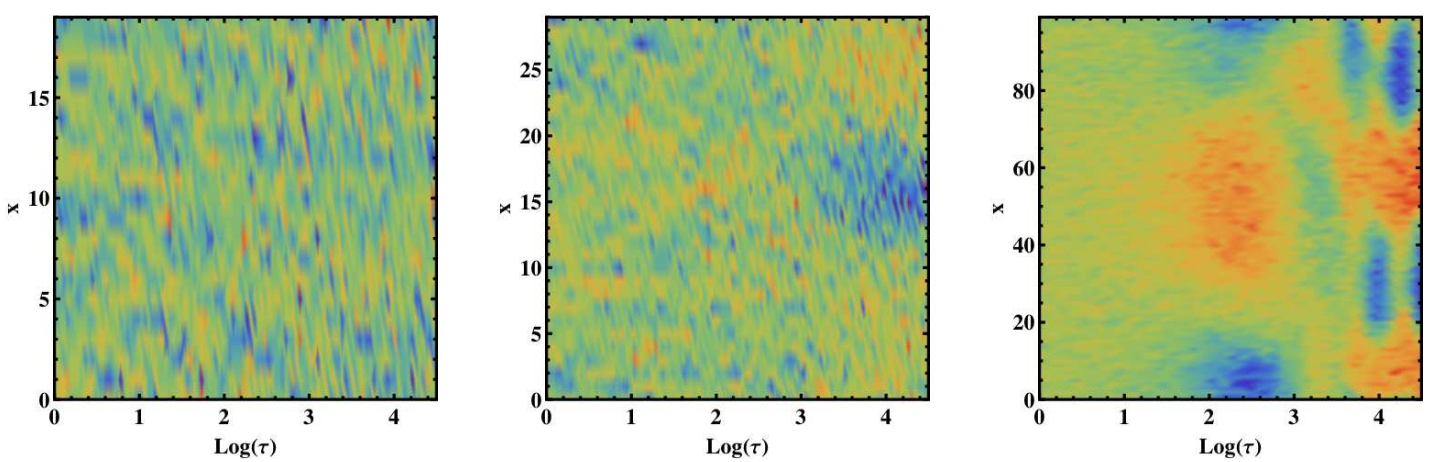

Fig. 9. (Color online) Space-time diagrams of the density fluctuations for the three perturbed systems, from left to right $L=20,30$ and 100. The color code shows in red the fluctuations above the average density, while in blue the fluctuations under the average density.
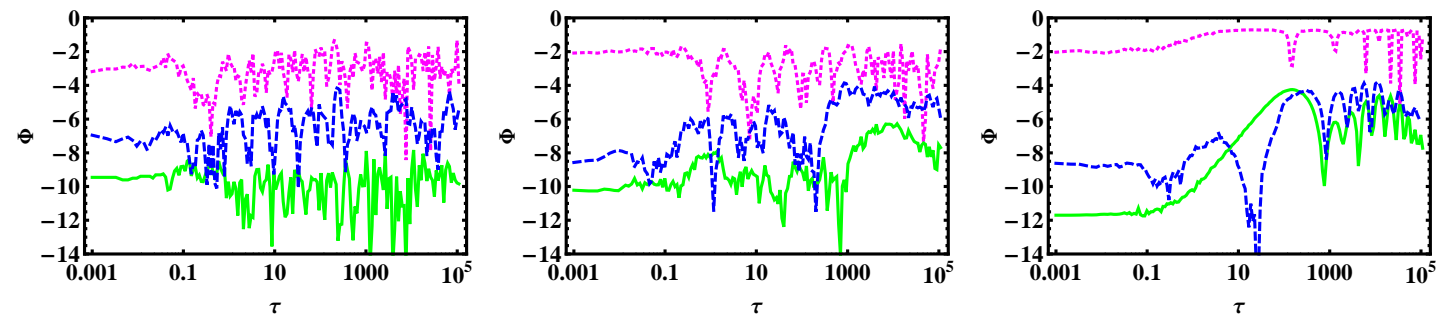

Fig. 10. (Color online) Time evolution for the first component of the normalized power spectra, $\Phi=\log \left(P\left(K_{m}\right)\right)$, in the three perturbed systems: from left to right $L=20,30$ and 100 . The magenta dotted line corresponds to the velocity, the dashed blue line to the temperature, and the solid green line to the density.
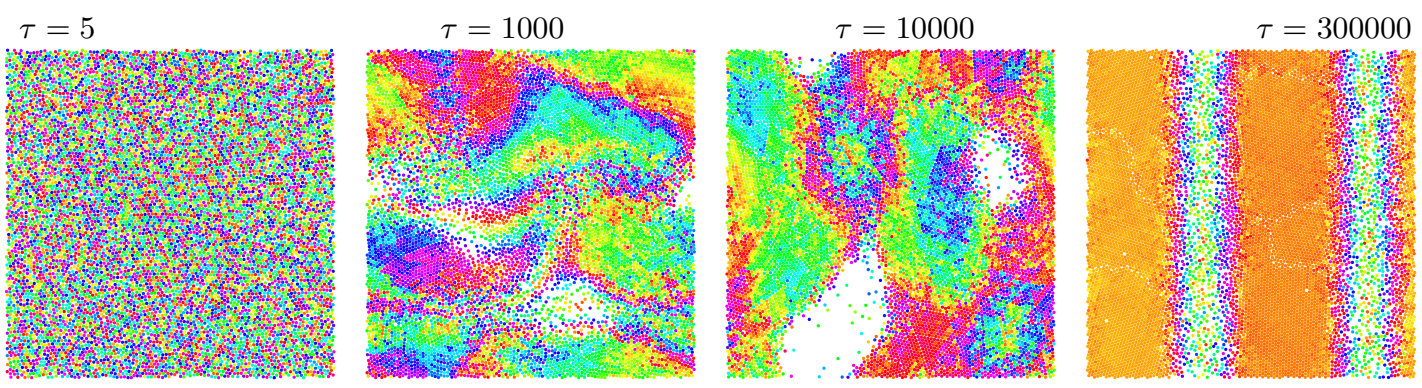

Fig. 11. (Color online) Four snapshots for the perturbed system with $L=100$ for times $\tau \simeq$ 5, 1000, 10000 and 300000. The particles are color coded depending on their relative kinetic energy normalized by the average. Red particles have more kinetic energy than the average, and blue particles have less.

\section{Conclusions}

In this paper different constitutive models for the pair correlation function at contact have been used to predict the onset of instability in a freely cooling 2D granular gas. First, the classical $g_{2}$ proposed by Henderson [27], second the $g_{\mathrm{pm}}$ by Grossman et al. [28], third the high density limit of the latter, $g_{\mathrm{fv}}[29]$, and finally, the $g_{Q}$ proposed by Luding in Refs. [22, 29].

Only the latter takes into account the disorder-order phase transition and the corresponding drop in the pressure around and above the critical density. This is also the one that results in the best fit with the data from ED simulations for mono-disperse disks.

While for low density the results do not depend on the choice of either $g_{2}$ or $g_{Q}$, the expected difference for the critical wave numbers at $\nu>0.75$ is rather small. The main result of this study 
is that around the crystallization density $(\nu \simeq 0.7)$, the instability of the sound mode - that may precede the clustering instability - is delayed, and a new mechanism of instability appears. The growth rate of the new mechanism is one order of magnitude slower than the classical heat mode. Even though we present theory and simulations for moderate dissipation, the theoretical predictions of this study are strictly valid for smaller dissipation only. Whether it is justified to neglect the density gradient contribution to heat flux has to be studied elsewhere.

For a given dissipation and density $\nu=0.7$, using $g_{Q}$, theory predicts that in order to become instable under clustering, the system size must be approximately $40 \%$ bigger than previously predicted by using $g_{2}$. We have found that for $\nu=0.7$ the best fit for Haff's cooling law is given by $g_{Q}$, and that a system with $L=20$ is stable to clustering, in contrast to the theory using $g_{2}$. These are clear differences and should be checked by more systematically examining the onset of the clustering instability at these densities, by using hard sphere simulations. The present results support the use of a modified pair-correlation function even though open questions remain.

Furthermore, we observe that the inclusion of modified constitutive models does not change the mechanism of the instability for small dissipation: the shear and the heat conduction modes are the principally unstable modes. Nevertheless, in the critical region, for different pair-correlation functions, the heat conduction mode is affected, while the shear mode is almost unchanged $(<10 \%)$. The use of the empirical pair-correlation function $g_{Q}$ predicts the appearance of a new mode in a small region around $\nu=0.7$. With the simulations realized here we were not able to excite this mode. Currently, we are studying different ways to accomplish this and find out to what extent the predictions of $g_{Q}$ are valid.

\section{Acknowledgments}

Helpful discussions with Sean McNamara are gratefully acknowledged. The hard work of the anonymous referee on the first version of this manuscript helped greatly to improve it. This study was supported by the Stichting voor Fundamenteel Onderzoek der Materie (FOM), financially supported by the Nederlandse Organisatie voor Wetenschappelijk Onderzoek (NWO), through the FOM project 07PGM27.

\section{References}

1. P. K. Haff. Grain flow as a fluid-mechanical phenomenon. J. Fluid Mech., 134:401-430, 1983.

2. I. Goldhirsch and G. Zanetti. Clustering instability in dissipative gases. Phys. Rev. Lett., 70(11):1619-1622, 1993.

3. S. McNamara. Hydrodynamic modes of a uniform granular medium. Phys. Fluids A, 5(12):3056 3070, 1993.

4. S. McNamara and W. R. Young. Dynamics of a freely evolving, two-dimensional granular medium. Phys. Rev. E, 53(5):5089-5100, 1996.

5. H. J. Herrmann, J.-P. Hovi, and S. Luding, editors. Physics of dry granular media - NATO ASI Series E 350, Dordrecht, 1998. Kluwer Academic Publishers.

6. S. Luding and H. J. Herrmann. Cluster growth in freely cooling granular media. Chaos, 9(3):673681, 1999.

7. T. Pöschel and S. Luding, editors. Granular Gases, Berlin, 2001. Springer. Lecture Notes in Physics 564.

8. J. J. Brey, M. J. Ruiz-Montero, and A. Dominguez. Shear state of freely evolving granular gases. Phys. Rev. E, 78(4):041301, 2008.

9. P. Richard, A. Valance, J. F. Metayer, P. Sanchez, J. Crassous, M. Louge, and R. Delannay. Rheology of confined granular flows: Scale invariance, glass transition, and friction weakening. Phys. Rev. Lett., 101(24):248002, 2008.

10. E. Khain. Bistability and hysteresis in dense shear granular flow. Europhys. Lett., 87:14001, 2009.

11. T. Shinbrot, N. H. Duong, M. Hettenbach, and L. Kwan. Coexisting static and flowing regions in a centrifuging granular heap. Granular Matter, 9(5):295-307, 2007. 
12. H. P. Zhu, Z. Y. Zhou, R. Y. Yang, and A. B. Yu. Discrete particle simulation of particulate systems: A review of major applications and findings. Chem. Eng. Science, 63(23):5728-5770, 2008.

13. I. Goldhirsch, M.-L. Tan, and G. Zanetti. A molecular dynamical study of granular fluids I: The unforced granular gas in two dimensions. Journal of Scientific Computing, 8:1-40, 1993.

14. J. Javier Brey, M. J. Ruiz-Montero, and D. Cubero. Homogeneous cooling state of a low-density granular flow. Phys. Rev. E, 54:3664-3671, 1996.

15. S. Luding. Clustering instabilities, arching, and anomalous interaction probabilities as examples for cooperative phenomena in dry granular media. T.A.S.K. Quarterly, Scientific Bulletin of Academic Computer Centre of the Technical University of Gdansk, 2(3):417-443, July, 1998.

16. S. Luding and S. McNamara. How to handle the inelastic collapse of a dissipative hard-sphere gas with the TC model. Granular Matter, 1(3):113-128, 1998. e-print cond-mat/9810009.

17. S. Miller and S. Luding. Cluster growth in two- and three-dimensional granular gases. Phys. Rev. E, 69:031305, 2004.

18. S. Luding. Structure and cluster formation in granular media. Pramana-Journal of Physics, 64(6):893-902, 2005.

19. B. D. Lubachevsky. How to simulate billards and similar systems. J. Comp. Phys., 94(2):255, 1991.

20. D. Bonamy, F. Daviaud, L. Laurent, M. Bonetti, and J. P. Bouchaud. Multiscale clustering in granular surface flows. Phys. Rev. Lett., 89(3):034301, 2002.

21. N. Sela and I. Goldhirsch. Hydrodynamic equations for rapid flows of smooth inelastic spheres to Burnett order. J. Fluid Mech., 361:41-74, 1998.

22. Stefan Luding. Towards dense, realistic granular media in 2d. Nonlinearity, 22(12):R101-R146, 2009.

23. J. T. Jenkins and M. W. Richman. Kinetic theory for plane shear flows of a dense gas of identical, rough, inelastic, circular disks. Phys. of Fluids, 28:3485-3494, 1985.

24. I. S. Aranson and L. S. Tsimring. Patterns and collective behavior in granular media: Theoretical concepts. Rev. Mod. Phys., 78(2):641-692, 2006.

25. K. Saitoh and H. Hayakawa. Rheology of a granular gas under a plane shear. Phys. Rev. E, 75(2):021302, 2007.

26. E. Khain. Hydrodynamics of fluid-solid coexistence in dense shear granular flow. Phys. Rev. E, 75(5):051310, 2007.

27. D. Henderson. A simple equation of state for hard discs. Molec. Phys., 30(3):971-972, 1975.

28. E. L. Grossman, T. Zhou, and E. Ben-Naim. Towards granular hydrodynamics in two-dimensions. Phys. Rev. E, 55:4200, 1997.

29. S. Luding. Global equation of state of two-dimensional hard sphere systems. Phys. Rev. E, 63:042201-1-4, 2001. 